\title{
Métrologie à SOLEIL. Des outills de mesure de la forme des miroirs synchrotron: un microscope interférométrique à balayage de phase et raccordement de champ
}

\author{
Muriel THOMASSET, Groupe Optique de SOLEIL \\ muriel.thomasset@synchrotron-soleil.fr
}

La cohérence des nouvelles sources synchrotron nécessite l'utilisation d'optiques limitées par la diffraction à des longueurs d'ondes inférieures au nanomètre. La fabrication des surfaces optiques a énormément progressé ces dernières années grâce aux techniques de polissage déterministe. Des surfaces avec des défauts de moins de $1 \mathrm{~nm}$ de hauteur pour des fréquences spatiales de l'ordre de $1 \mathrm{~mm}$ peuvent être actuellement produites. Le synchrotron SOLEIL ne possède pas le moyen de produire de telles optiques mais se doit d'être capable de les contrôler avant leur montage sur les lignes de lumière.

e profilomètre optique du laboratoire Lde métrologie du groupe Optique (long trace profiler) a longtemps été la référence en termes de contrôle de forme des surfaces et de mesure de rayons de courbure. Le polissage déterministe nécessite dorénavant une mesure bidimensionnelle des surfaces avec une meilleure sensibilité, bien en dessous du nm Ces exigences de sensibilité et de résolution spatiale nous ont naturellement orientés vers la microscopie interférométrique à balayage de phase. Un objectif interférométrique télécentrique permet, quant à lui, de résoudre les problèmes de distorsion des franges et de profondeur de champ. Les miroirs synchrotrons pouvant atteindre plusieurs centaines de $\mathrm{mm}$ de longueur, la reconstruction de la surface totale du miroir se fait par raccordement des différents champs de mesure (typiquement de $10 \mathrm{~mm}$ de diamètre) effectués avec le recouvrement adéquat (figure 1).

\section{L'instrument}

L'instrument (figure 2) a été étudié et construit en collaboration avec les sociétés MBO-Metrology (Wissous, Essonne) et

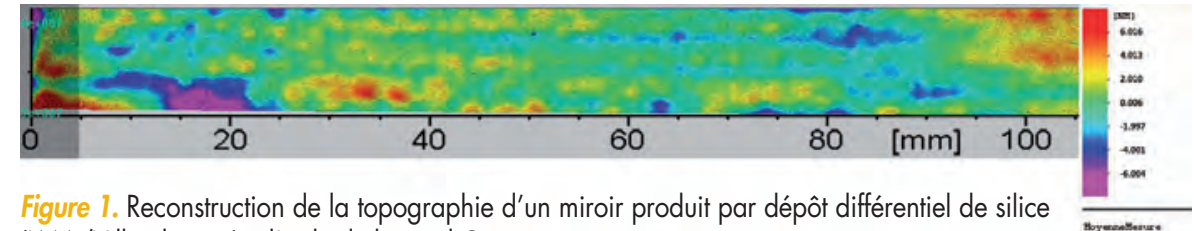
(LMA/Villeurbanne) à l'aide du logiciel Optocat.

EOTECH (Marcoussis, Essonne). La société MBO-Metrology a effectué le dessin et le calcul de l'optique. L'interféromètre utilise le principe du Michelson travaillant à différence de marche nulle ce qui nous permet d'utiliser une source légèrement polychromatique pour faciliter la recherche de la position de mise au point et s'affranchir des problèmes de speckle. La source retenue est de type LED émettant à $505 \mathrm{~nm}$ et équipée d'un filtre de $10 \mathrm{~nm}$ de large. Le champ observable est de $16 \times 12 \mathrm{~mm}^{2}$ soit un grandissement de 2,5. L'instrument a été construit par la société EOTECH. Il est monté sur une structure rigide en granite, et installé dans une zone à température, hygrométrie et empoussièrement contrôlés. Le logiciel de contrôle et d'acquisition est aussi développé par EOTECH. Il est modulaire et flexible ce qui correspond bien à nos besoins de développement. Ce principe de modularité a aussi été utilisé pour le design mécanique et optique : chaque partie de l'instrument est facilement interchangeable. La répétabilité actuelle de I'instrument est de $0.5 \mathrm{~nm}$ PV. Il est difficile d'en déduire les performances ultimes ; celles-ci sont en effet notablement influencées par les méthodes de traitement des interférogrammes enregistrés et particulièrement par la procédure de raccordement de champ.

\section{Le raccordement des champs de mesure : le "stitching 》}

La procédure de stitching est bien évidemment sensible aux erreurs de mesure, mais aussi à la distorsion d'image et à l'estimation de la surface de référence interférométrique. Dans une procédure pas à pas, ces défauts se propagent et s'amplifient à chaque raccordement d'un 
nouveau champ de mesure. Cependant dans une approche globale qui considère que la référence interférométrique est constante il est possible, avec un recouvrement suffisant des mesures, d'extraire la surface de référence de l'instrument et de reconstruire la topographie de la surface du miroir. Concrètement, différentes méthodes de stitching sont possibles et celle développée à SOLEIL est basée sur une méthode de maximum de vraisemblance. La figure 3 montre la comparaison entre différents algorithmes. Le premier, propagatif, proposé dans le logiciel Optocat de la société EOTECH ; le deuxième, $\mathrm{MBSI}$, proposé par la société $\mathrm{MBO}$ Metrology ; et le troisième, développé par le Groupe optique de SOLEIL. Les résultats des différentes méthodes sont comparés, à la figure 3, à une mesure de profil effectué au LTP. Celle-ci sert de référence, bien que la résolution spatiale de l'instrument ne soit que de $0,5 \mathrm{~mm}$, car celui-ci a bénéficié de nombreuses calibrations croisées. On remarque que les différents algorithmes donnent des résultats similaires

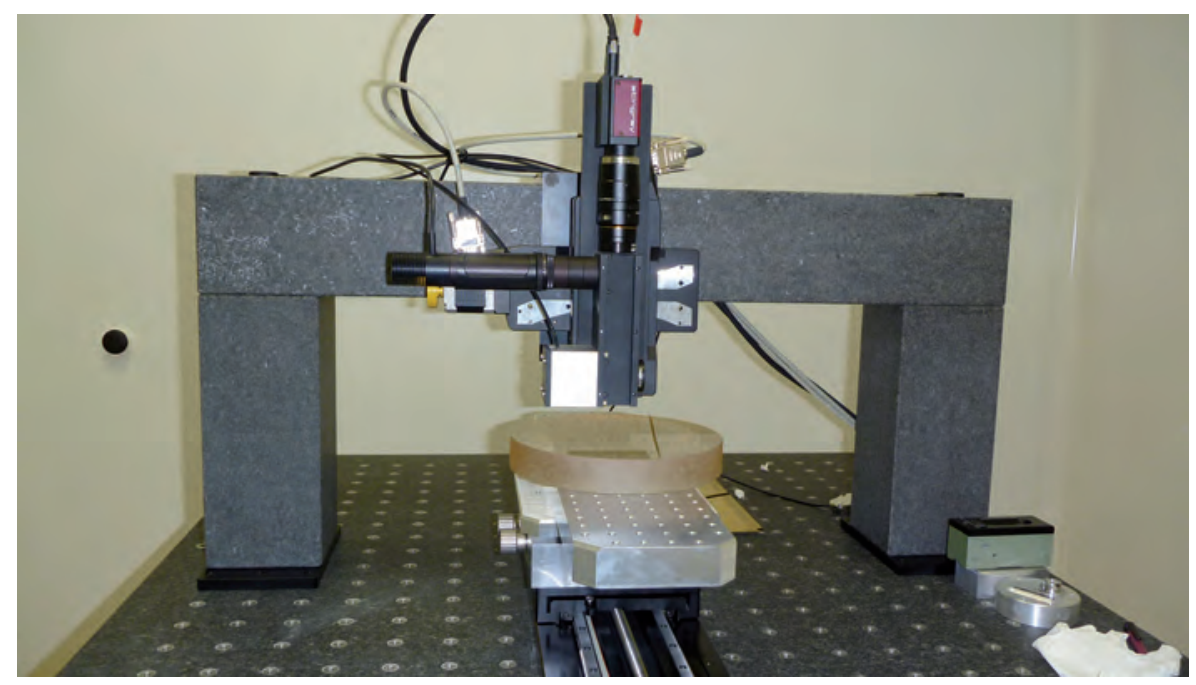

Figure 2. L'instrument NanoPro.

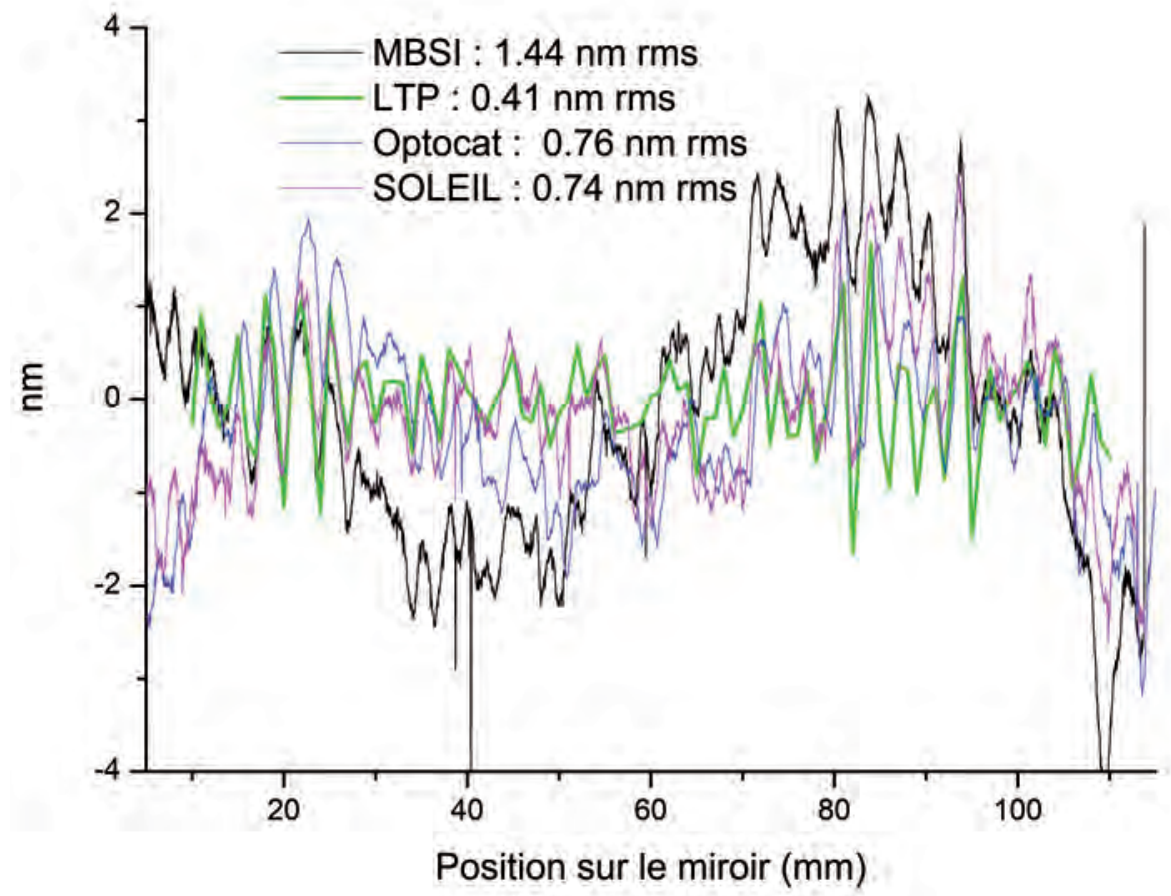

Figure 3. Comparaison de différents algorithmes de stitching.

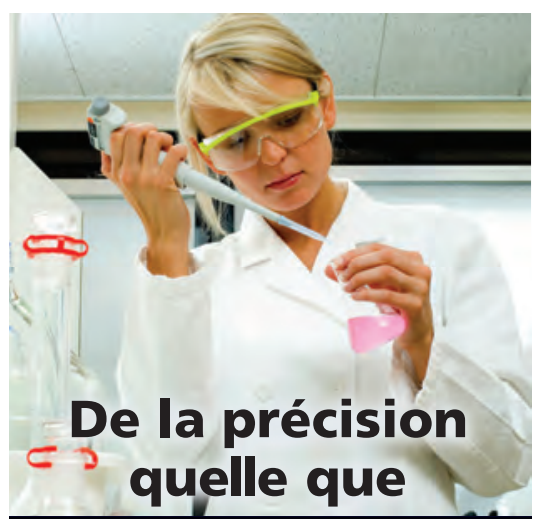

\section{soit la luminosité}

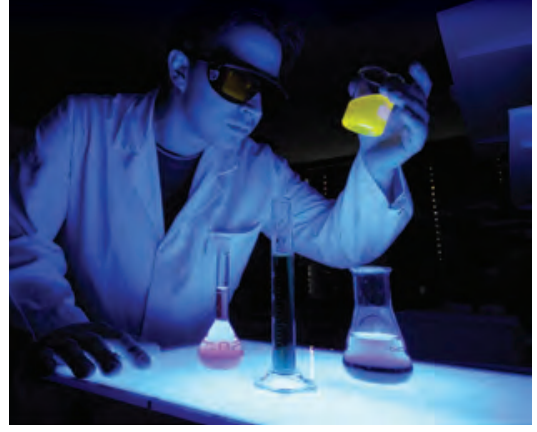

Le Maya LSL Spectromètre Ocean Optics Haute Sensibilité

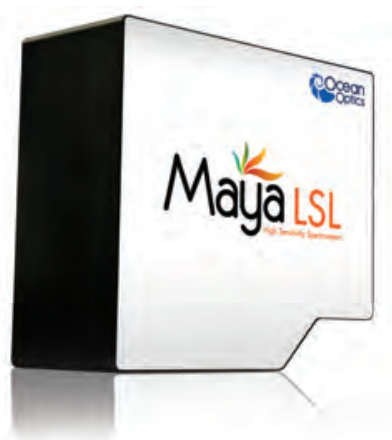

Quelles que soient vos conditions de laboratoire, par sa sensibilité le Maya LSL détecte des changements infimes rapidement et de manière fiable.

\section{Contactez nos} ingenieurs maintenant.

Tel: 0296054020 info@idil.fr www.idil.fr

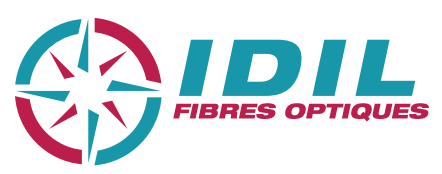




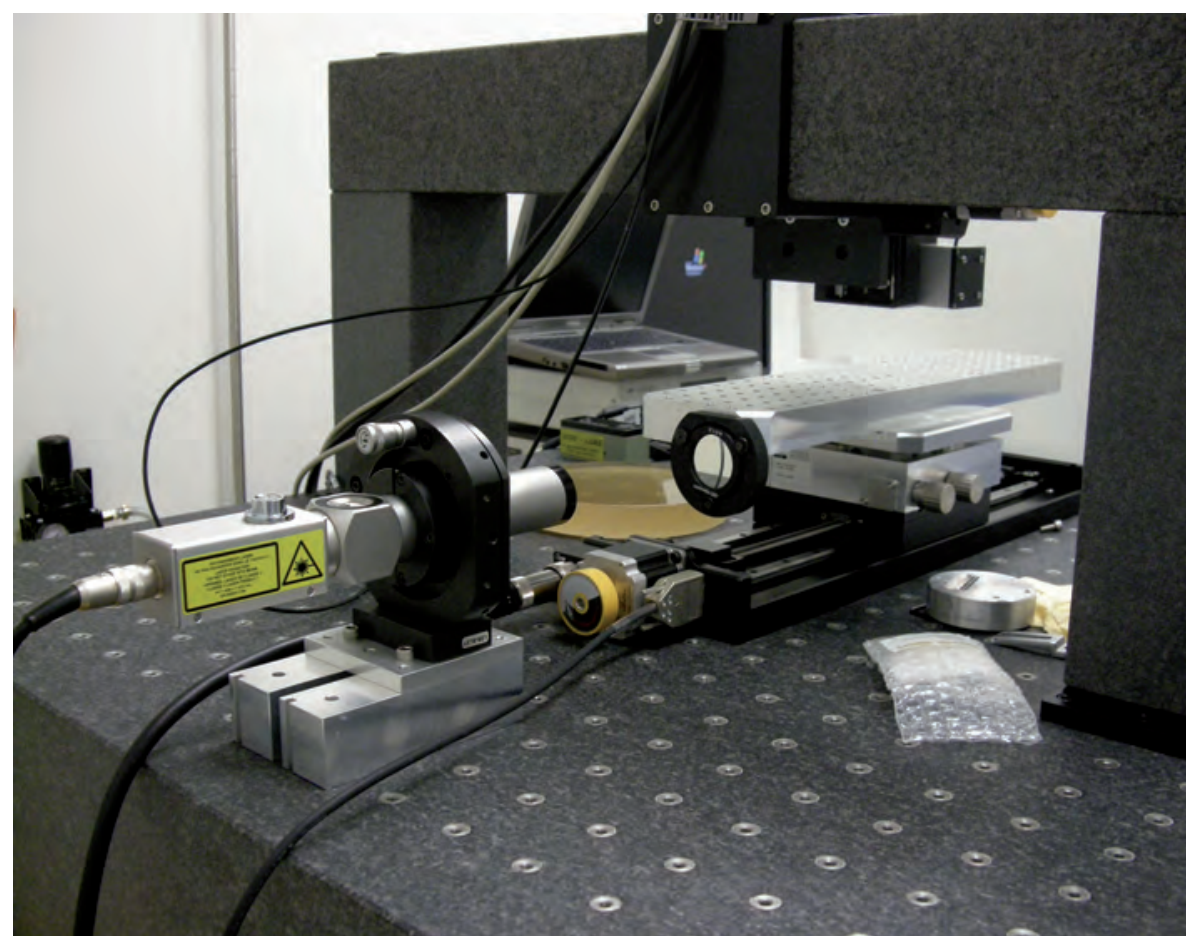

Figure 4. Lunette autocollimatrice placée à l'arrière de l'instrument qui enregistre les défauts angulaires de la platine de translation sur laquelle est placé le miroir à mesurer.

à une oscillation résiduelle du quatrième ordre près, plus ou moins prononcée. La distorsion du système optique, très faible, a été contrôlée et mise hors de cause. Les oscillations proviennent en réalité d'une estimation incorrecte, par les différents algorithmes, des orientations successives de la surface à chaque pas de translation. Depuis peu, une lunette autocollimatrice (figure 4) est placée à l'arrière de l'instrument et enregistre les angles de roulis et lacet de la platine. Dans une prochaine étape, on intégrera ces mesures aux algorithmes de reconstruction.

\section{Conclusion}

Un nouveau microscope à balayage de phase utilisant un objectif interférométrique télécentrique a été développé à SOLEIL en collaboration avec les sociétés EOTECH et MBO-Metrology. Une précision inférieure au $\mathrm{nm}$ a été atteinte. Différents algorithmes de raccordement de champs ont été testés dont un développé à SOLEIL. Cet instrument a été pensé comme un instrument de recherche offrant la possibilité de modifier la structure mécanique, le design optique et le pilotage informatique.

\section{Références}

Thomasset, M., Idir, M., Polack, F., Bray, C., Servant, C., A new Phase-shift Microscope Designed for High Accuracy Stitching Interferometry, Nuclear Instruments and Methods A, 2013, 710: 7-12

Polack, F. \& Thomasset, M., Determination and compensation of the "reference surface" from redundant sets of surface measurements, Nuclear Instruments and Methods A, 2013, 710: 67-71

\section{Remerciements}

Ce travail a bénéficié d'un financement de la Région lle de France SESAME 2007, convention \# F-08-1097 et de l'Agence Nationale de la Recherche, ANR 09-NANO-008-AXOC.

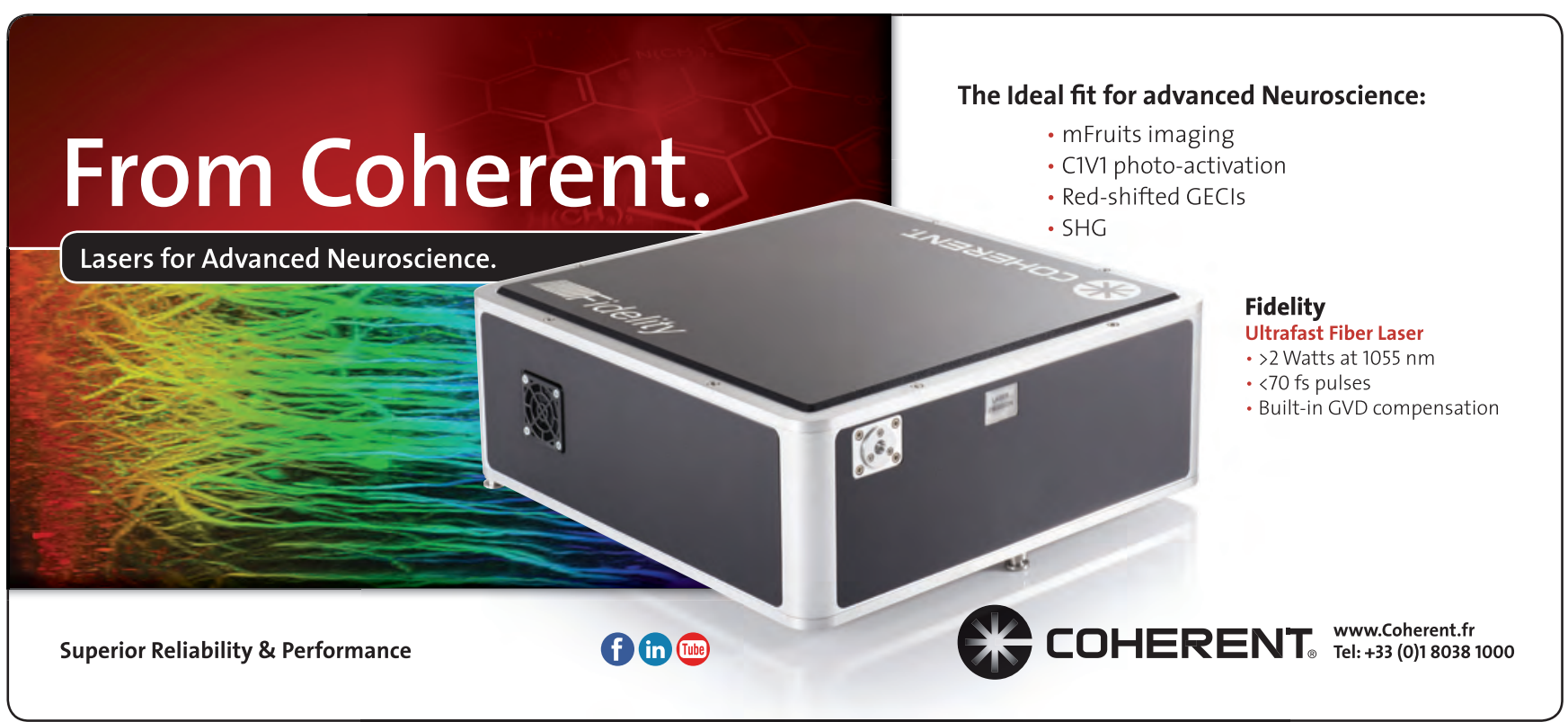

